Brésil : Espirito Santo (coll. PIC).

Diffère du précédent par la forme plus étroite, la coloration plus claire, les impressions basales du prothorax et la sculpture des élytres.

\title{
Description d'un Oxypoda nouveau de France
}

\author{
(Col. Staphylinidae)
}

\section{par J. Sainte-Claire Deville.}

Oxypoda (s. str.) Falcozi, n. sp. - O. longipedi Muls. et Rey affinis et simillima; ab eadem statura evidenter crassiore antennisque multo brevioribus optime distinguenda. - Long. 4,כ̆ mill.

Patria: Alpes Galliae, in Arctomyos alpini nidis subterraneis.

Extrêmement voisin des 0 . vittata Märk. et longipes Muls. et Rey, ce nouveau Staphylinide est identique à la dernière de ces deux espèces pour la conformation des tarses postérieurs. Il s'en rapproche assez pour qu'il soit superflu d'en donner une description complète.

Examiné de côté, l'O. Falcozi est beaucoup moins aplati que le longipes; son épaisseur égale à peu près celle de l'Aleochara puberula Klug, avec lequel il présente une ressemblance superficielle. Comparées à celles de l'o. longipes, les antennes sont beaucoup plus courtes et plus épaisses; les $2^{\mathrm{e}}$ et $3^{\mathrm{e}}$ articles sont égaux, les $4^{\mathrm{e}}$ et $\breve{\mathrm{e}}^{\mathrm{e}}$ obconiques et à peu près aussi longs que leur largeur moyenne, les suivants jusqu'au $10^{e}$ franchement transverses; le dernier article n'est pas comprimé. Le pronotum est proportionnellement plus grand et plus brillant, les élytres un peu plus courts; l'abdomen, sensiblement moins atténué vers l'extrémité, est un peu plus brillant.

L'O. Falcozi a été découvert par notre collègue M. Falcoz, de Vienne (Isère), en examinant de la litière recueillie au fond des terriers de la Marmotte des Alpes (Arctomys alpinus L.) et provenant des environs de Briançon (H $\left.{ }^{\text {tes }}-A l p e s\right)(')$.

(1) On sait que l'espèce la plus voisine, O. longipes $\mathrm{Muls}$. et $\mathrm{Rey}$, vit à peu près exclusivement dans les nids de taupes. Les $O$. viltata M ärk. et O. spectabilis Mä rk., plus éclectiques, ont étè à la fois observés dans les fourmilières, les nids souterrains de 'Guêpes et de Bourdons, des terriers de lapins, etc. 


\section{$2 \mathrm{BHL}$ Biodiversity Heritage Library}

1913. "Description d'un Oxypoda nouveau de France." Bulletin de la Société entomologique de France 1913, 134-134.

https://doi.org/10.5962/bhl.part.16276.

View This Item Online: https://www.biodiversitylibrary.org/item/36377

DOI: https://doi.org/10.5962/bhl.part.16276

Permalink: https://www.biodiversitylibrary.org/partpdf/16276

\section{Holding Institution}

Smithsonian Libraries

\section{Sponsored by}

Smithsonian

\section{Copyright \& Reuse}

Copyright Status: NOT_IN_COPYRIGHT

This document was created from content at the Biodiversity Heritage Library, the world's largest open access digital library for biodiversity literature and archives. Visit BHL at https://www.biodiversitylibrary.org. 